\section{Coexisting vascular lesions in reversible cerebral vasoconstriction syndrome} cerebral vasoconstriction syndrome

\author{
M Akif Topcuoglu', ${ }^{1,2}$ Oguzhan Kursun ${ }^{1,3}$ and Aneesh B Singhal'
}

\section{Cephalalgia}

2017, Vol. 37(I) 29-35

(C) International Headache Society 2016 Reprints and permissions: sagepub.co.uk/journalsPermissions.nav DOI: $10.1177 / 0333102416637826$ cep.sagepub.com

@SAGE

\begin{abstract}
Background: The pathophysiology of reversible cerebral vasoconstriction syndrome (RCVS) is not known. Published cases have documented coexisting cervical artery dissection and unruptured aneurysms, raising the possibility that ultrastructural vessel wall abnormalities underlie the development of vascular lesions as well as RCVS.

Methods: In this retrospective study we compared the frequency of neurovascular abnormalities in 158 consecutive RCVS patients, 44 patients with primary angiitis of the central nervous system (PACNS, positive controls), and 177 nonstroke patients with acute neurological symptoms (non-arteriopathy controls).

Results: Coexisting neurovascular abnormalities were significantly higher $(p<0.00 \mathrm{I})$ in RCVS $(23 \%)$ as compared to the PACNS $(5 \%)$ or non-arteriopathy groups (8\%). Cervical artery dissections were noted only in the RCVS group $(8 \%$, $p<0.001$ ). The RCVS group had more unruptured aneurysms than PACNS (I3\% vs. $5 \%, p=0.099)$ or non-arteriopathy controls ( $13 \%$ vs. $7 \%, p=0.05$ ). Seven RCVS patients also had other vascular malformations (venous anomaly, cavernous malformations, fibromuscular dysplasia). There was no significant association between coexisting vascular abnormalities and brain lesions or discharge clinical outcome in the RCVS group.

Conclusion: The high prevalence and heterogeneous anatomy of coexisting vascular lesions suggest that subtle ultrastructural arterial wall abnormalities may contribute to their development and also predispose to RCVS.
\end{abstract}

\title{
Keywords
}

Cerebral vasoconstriction, cerebral vasculitis, thunderclap headache, aneurysm, cervical artery dissection, vascular malformation

Date received: 13 October 2015; accepted: 6 February 2016

\section{Introduction}

Reversible cerebral vasoconstriction syndrome (RCVS) is characterized by recurrent thunderclap headaches and reversible cerebral arterial vasoconstriction, often with ischemic or hemorrhagic strokes (1-5). An expanding spectrum of conditions that have overlapping clinical, imaging, or angiographic features with RCVS have been identified including posterior reversible encephalopathy syndrome (PRES), migraine, and primary thunderclap headache $(\mathrm{TCH})$.

Arterial histopathology and electron microscopy studies in RCVS patients have shown no abnormalities (6). However, recent publications have documented a high incidence of cervical artery dissection in patients with RCVS (7-9). Some RCVS patients harbor unruptured saccular aneurysms $(4,10,11)$. This suggests that hitherto unidentified structural abnormalities or perhaps non-structural abnormalities of vessel tone predispose to both RCVS and the development of vascular lesions. In this study, we compared the frequency of coexisting cerebral vascular abnormalities in patients with RCVS, primary angiitis of the central nervous system (PACNS, positive controls), and individuals who underwent head/neck computerized tomography angiography (CTA) for evaluation of acute nonstroke neurological symptoms (negative controls).

\footnotetext{
'Department of Neurology, Massachusetts General Hospital, USA ${ }^{2}$ Department of Neurology, Hacettepe University Hospitals, Turkey ${ }^{3}$ Neurology Clinics, Ankara Numune Education and Research Hospital, Turkey
}

\section{Corresponding author:}

Aneesh B Singhal, Department of Neurology, ACC-729C, Massachusetts General Hospital, 55 Fruit Street, Boston, MA 02।I4, USA.

Email: asinghal@partners.org 


\section{Methods}

This retrospective study was approved by our hospital's Human Research Committee.

\section{Patients}

The RCVS sample comprised 158 consecutive patients personally encountered at Massachusetts General Hospital (MGH) from 1998 to 2015. This group included 83 of the 84 patients whose clinical and brain imaging features were published in a collaborative study (one patient who did not undergo angiography was excluded) (1). The diagnosis of RCVS was based on key features published in 2007 (2), which have been used in multiple cohort studies $(1,3-5,12)$.

To investigate whether RCVS patients have a high frequency of coexisting vascular abnormalities, we assembled two control groups: PACNS (positive control) and "non-arteriopathy" (negative control). The PACNS group was extracted from three sources: personally encountered cases from 1998 to 2015; patients with discharge International Classification of Diseases, ninth edition (ICD-9) diagnostic code 437.4 (vasculitis) from 1993 onward; and patients undergoing brain biopsy for suspected cerebral vasculitis from 2002 to 2015. Of the 47 patients retrieved, for this analysis we included the 44 patients who underwent cerebral angiographic evaluation. PACNS was confirmed by brain biopsy or autopsy in $18(41 \%)$ and the rest had progressive neurological deficits with inflammatory cerebral arteriopathy and no evidence of infection (e.g. varicella zoster, Lyme) or systemic vasculitis (e.g. systemic lupus erythematosus, polyarteritis) (13).

The non-arteriopathy control group comprised 177 patients with a non-stroke diagnosis from a dataset of
462 consecutive patients who underwent head and neck CTA in our emergency department over a span of eight months in 2003, for evaluation of neurological symptoms. These 177 patients comprised 37 with nonthunderclap headache, 30 with transient neurological symptoms without radiological infarction, 24 with trauma, 15 with seizure, 13 with syncope, nine with vertigo, eight with delirium, seven with neck pain, five with infectious and/or demyelinating diseases, and 22 with other miscellaneous conditions.

\section{Vascular imaging}

Many patients had more than one vascular imaging modality. In order of priority, we analyzed the clinical radiology reports of digital subtraction angiography (DSA), CTA, and lastly magnetic resonance angiography (MRA). In the RCVS group, analysis was based on DSA findings in 63, CTA in 77, and MRA in 18 patients. In the PACNS group, analysis was based on DSA findings in 32, CTA in five, and MRA in seven patients. In the non-arteriopathy group, analysis was based on CTA for all patients; these patients did not undergo initial DSA.

\section{Brain MRI}

To investigate for vascular lesions such as cavernomas that may not be apparent on angiography, we reviewed the reports of brain magnetic resonance imaging (MRI) scans, if performed, in all patients. Brain MRI was performed in 147 RCVS patients (with contrast in 103); 44 PACNS patients (all with contrast); and 86 nonarteriopathy controls (46 with contrast). Further, we reviewed the brain scans of all RCVS patients and

Table I. Coexisting vascular findings in RCVS, PACNS and control groups.

\begin{tabular}{|c|c|c|c|c|c|c|}
\hline & $\begin{array}{l}\text { RCVS } \\
(n=158)\end{array}$ & $\begin{array}{l}\text { PACNS } \\
(n=44)\end{array}$ & $\begin{array}{l}\text { Control } \\
(n=177)\end{array}$ & $p$ value $^{a}$ & $\begin{array}{l}p \text { value }^{b} \\
\text { RCVS vs PACNS }\end{array}$ & $\begin{array}{l}P \text { value }^{\mathrm{b}} \\
\text { RCVS vs control }\end{array}$ \\
\hline Gender (female, \%) & I 24 (78\%) & $13(30 \%)$ & $84(47 \%)$ & $<0.001$ & $<0.001$ & $<0.001$ \\
\hline Age $($ mean $\pm S D)$ & $44 \pm 13$ & $52 \pm 16$ & $58 \pm 21$ & $<0.00 \mathrm{I}$ & 0.001 & $<0.001$ \\
\hline Any vascular lesion & 37 (23\%) & $2(5 \%)$ & 14 (8\%) & $<0.001$ & 0.002 & $<0.001$ \\
\hline Unruptured saccular aneurysm & $20(13 \%)$ & $2(5 \%)$ & $12(7 \%)$ & 0.116 & 0.099 & 0.05 \\
\hline Aneurysm size $>2 \mathrm{~mm}$ & $12(8 \%)$ & $0(0 \%)$ & $6(3 \%)$ & 0.068 & 0.048 & 0.07 \\
\hline Cervical artery dissection & $13(8 \%)$ & $0(0 \%)$ & $0(0 \%)$ & $<0.001$ & 0.04 & $<0.001$ \\
\hline Other vascular malformation ${ }^{c}$ & $5(3 \%)$ & $0(0 \%)$ & I (I\%) & 0.173 & 0.29 & 0.19 \\
\hline Fibromuscular dysplasia & $2(1 \%)$ & $0(0 \%)$ & I (I\%) & 0.727 & 0.61 & 0.46 \\
\hline
\end{tabular}

${ }^{a}$ ANOVA was used for age; Fisher-Freeman-Halton tests were used for the remaining.

'Student's $t$-test was used for age; Fisher's exact test was used for others.

'Other vascular malformations include arteriovenous malformation, developmental venous anomaly and cavernous malformations.

RCVS: reversible cerebral vasoconstriction syndrome; PACNS: primary angiitis of the central nervous system; ANOVA: analysis of variance. 
classified the findings as normal (no intracranial lesion), infarct, parenchymal hemorrhage, subarachnoid hemorrhage, edema (PRES), or combinations.

\section{Statistical analysis}

Analysis of variance (ANOVA), Wilcoxon and Student's $t$ tests were used for continuous variables. Chi-square, Fisher exact, and Fisher-Freeman-Halton tests were used for categorical variables. No adjustments were made for multiple comparisons. Linear and logistic regression analyses were performed to identify independent predictors of vascular abnormalities. A $p<0.05$ was considered significant. SPSS version 21 was used for analyses.

\section{Results}

The RCVS group was significantly younger and had a higher proportion of women as compared to the other groups (Table 1). A significantly higher number of RCVS patients proved to have coexisting cerebral vascular abnormalities as compared to controls, either separately ( $p=0.002$ versus PACNS; $p<0.001$ versus non-arteriopathy controls, Table 1$)$, or in combination $(23 \%$ versus $7 \%, p<0.001)$.
Cervical artery dissections were detected only in the RCVS group (8\%); 10 of the 13 RCVS patients with dissection had single-artery dissection including seven affecting the extracranial internal carotid artery (ICA, Figure 1) and three in the extracranial vertebral artery. Three RCVS patients had bilateral ICA dissections; one had an additional vertebral artery dissection. Of the total 17 dissected arteries, 11 were on the left and six on the right side. All dissections, except for two ICA dissections occurring in the same patient, were nonocclusive. All dissections were acute, diagnosed by DSA in six, CTA in five and MRA in two patients. All dissections improved or resolved on follow-up and their management was variable with the use of antiplatelet agents, anticoagulants, or neither.

Unruptured cerebral saccular aneurysms were detected in all three groups (Figure 2). Their size ranged from $1 \mathrm{~mm}$ to $12 \mathrm{~mm}$. Table 1 shows the frequency of aneurysms measuring $>2 \mathrm{~mm}$ (i.e. definite aneurysms rather than infundibular outpouchings) across groups. All aneurysms, as well as aneurysms $>2 \mathrm{~mm}$, were more frequent in RCVS patients as compared to PACNS and non-arteriopathy controls, either alone (Table 1) or in combination (any size, $13 \%$ versus $6 \%, p=0.027$; more than $2 \mathrm{~mm}, 8 \%$ versus $3 \%, p=0.026$ ). The RCVS group had a total of 26 aneurysms, including six patients with

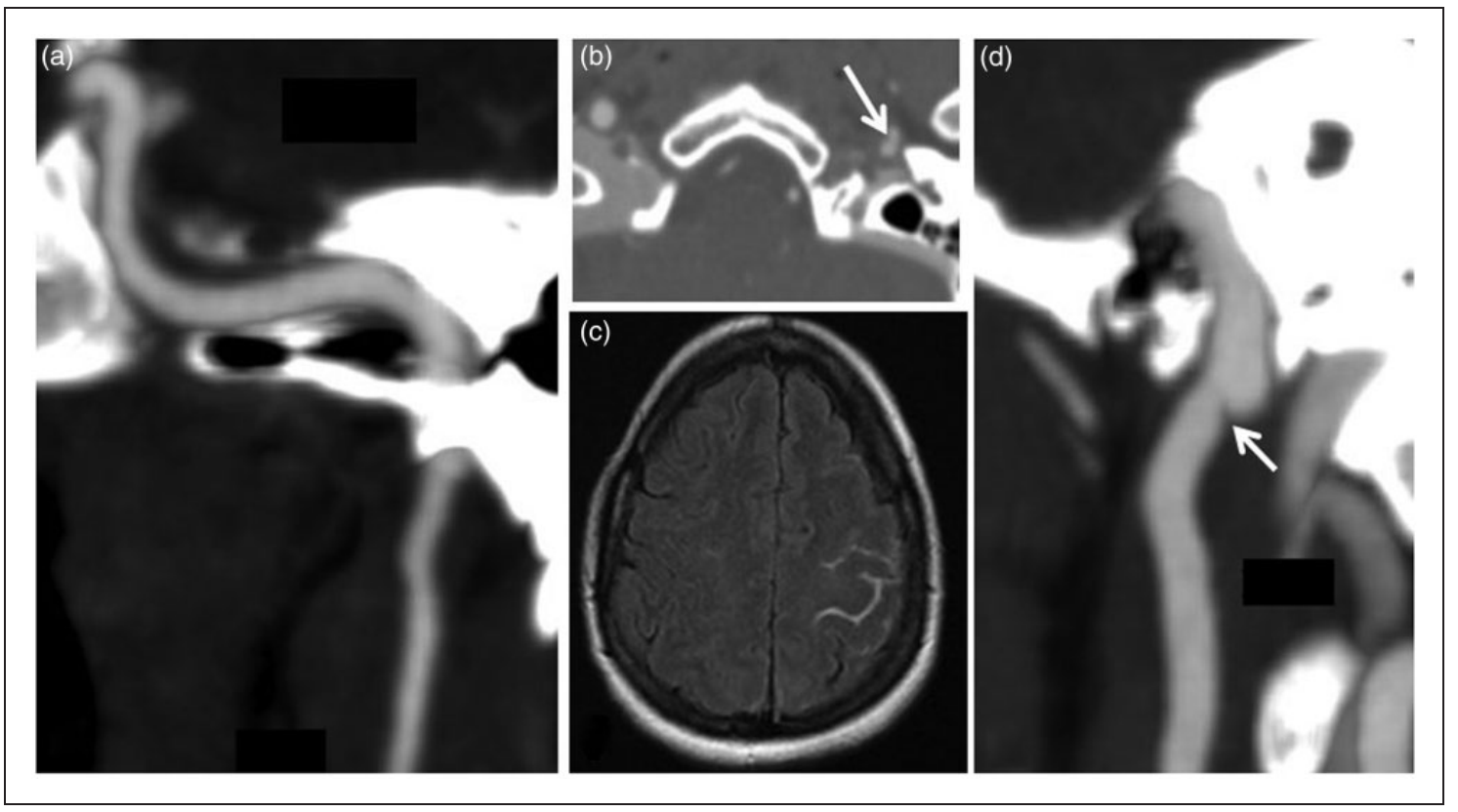

Figure I. Imaging findings in a 39-year-old woman with post-partum RCVS. Admission CTA shows left extracranial carotid artery dissection on coronal (a) and axial ((b), arrow) maximum intensity projection images. (c) MRI (axial fluid-attenuated inversion recovery image) shows convexal subarachnoid hemorrhage. (d) Follow-up CTA after six weeks shows a persistent dissection flap (arrow) but improvement of luminal flow.

RCVS: reversible cerebral vasoconstriction syndrome; CTA: computed tomography angiography; MRI: magnetic resonance imaging. 


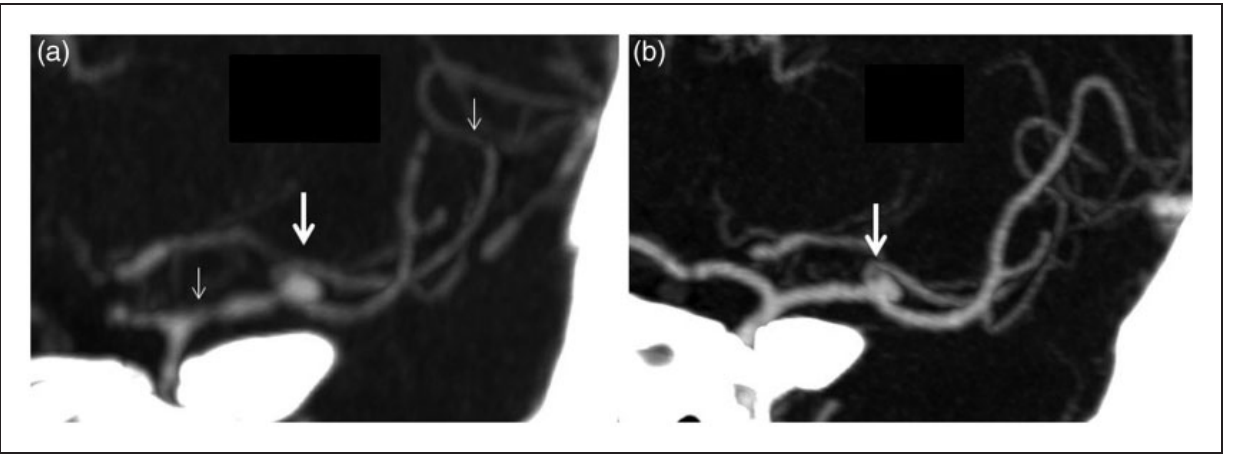

Figure 2. CTA maximum intensity projection images in a 35-year-old woman with RCVS associated with vasoconstrictive drugs. (a) Admission scan shows segmental cerebral arterial vasoconstriction and a 4-mm saccular aneurysm at the bifurcation of the middle cerebral artery. (b) Follow-up scan after three weeks shows resolution of vasoconstriction and persistence of the aneurysm. CTA: computed tomography angiography; RCVS: reversible cerebral vasoconstriction syndrome.

two aneurysms each. They were located in the intracranial ICA (infra-/supraclinoid, paraopthalmic, superior hypophyseal, terminus) in 13 , cavernous ICA in four, middle cerebral artery bifurcation in three, posterior cerebral artery in two, and one each in the anterior communicating artery, distal vertebral artery, posterior communicating artery, and anterior choroidal artery. The PACNS group showed a total of three aneurysms with one patient harboring two aneurysms; all measured $<2 \mathrm{~mm}$ and they were located in the posterior communicating artery, origin of the posterior inferior cerebellar artery, and cavernous ICA. The non-arteriopathy group showed a total of 14 aneurysms with two patients harboring two aneurysms; their size ranged from $1 \mathrm{~mm}$ to $12 \mathrm{~mm}$; four were located in the supra-/paraclinoid or terminal ICA; three in the anterior communicating artery; two in the middle cerebral artery bifurcation; two in the intracranial vertebral artery (one fusiform); and one each in the posterior inferior cerebellar artery, superior cerebellar artery, and cavernous ICA.

A variety of other coexisting vascular lesions were detected. Persistent (irreversible) accordion-shaped irregularities in the extracranial ICAs, consistent with fibromuscular dysplasia, were observed in two RCVS patients (both with dissection). On brain MRI, the RCVS group showed heterogeneous vascular abnormalities: one cerebellar venous angioma, one capillary telangiectasia, two developmental venous anomalies, and two cavernous malformations (Figure 3). The PACNS group showed no vascular abnormalities on brain MRI. One non-arteriopathy patient had a cerebellar venous anomaly and another had a left middle cerebral artery fusiform dilation.

In analyses restricted to CTA alone, vascular abnormalities were detected in $21 \%$ RCVS, $8 \%$ controls, $0 \%$ PACNS $(p=0.009)$. In analyses restricted to individuals with MRI, the rate of lesions on vascular/parenchymal imaging was significantly higher in
RCVS as compared to PACNS or controls (25\% versus $4 \%$ and $9 \%, p<0.001)$. Regression analyses including age, gender, brain MRI, and vascular imaging modality showed that RCVS remained a significant predictor of any vascular abnormality $(p=0.004$, odds ratio (OR) 3.261 , 95\% confidence interval (CI) 1.455-7.305) and tended to predict unruptured aneurysms ( $p=0.066$, OR $2.258,95 \%$ CI $0.939-6.972$ ).

We investigated the relationship between vascular abnormalities and brain lesions in the RCVS group. Brain imaging was normal in 25\%; $36 \%$ showed convexal subarachnoid hemorrhages, $14 \%$ showed parenchymal hemorrhages, 34\% showed infarctions, and $30 \%$ showed edematous (PRES) lesions. Lesion combinations were present in $28 \%$. The discharge modified Rankin scale (mRS) score was $>3$ in only $9.5 \%$, including four deaths. There was no association between coexisting vascular abnormalities and RCVS lesions. There was no significant difference in the mean mRS score between RCVS patients with and without vascular lesions.

\section{Discussion}

In this study we compared the frequencies of coexisting vascular abnormalities in patients with RCVS, PACNS, and patients with neurological symptoms without stroke. The RCVS group had a significantly higher rate of vascular abnormalities as compared to the other groups. The PACNS group showed no significant differences as compared to non-arteriopathy controls. These observations may have implications for the pathophysiology of RCVS.

An association between RCVS and cervical artery dissection has been noted in prior case reports $(7,9)$ and case series (8). In a French study, $12 \%$ of 173 patients with RCVS had cervical artery dissection, and $7 \%$ of 285 patients with cervical artery dissection 


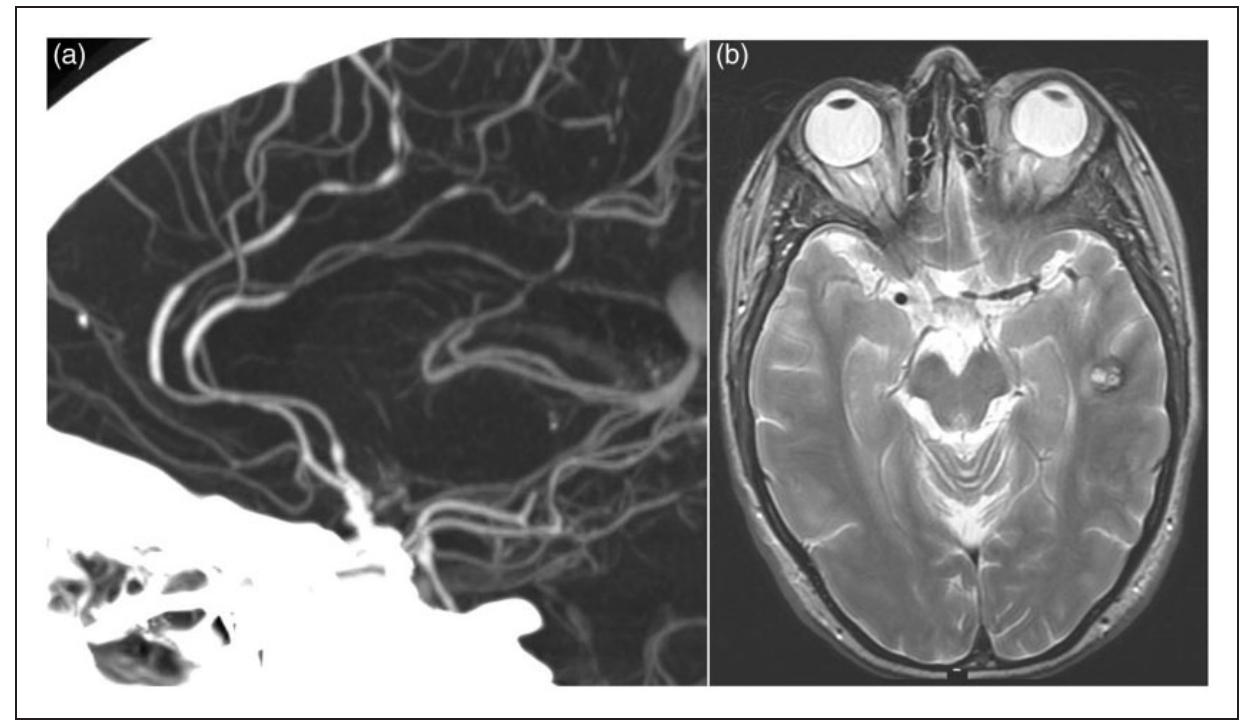

Figure 3. Imaging findings in a 43-year-old woman with RCVS associated with sexual orgasm. (a) CTA maximum intensity projection image shows the classic "sausage on a string" appearance of intracerebral arteries. (b) MRI (axial fluid-attenuated inversion recovery image) shows an asymptomatic cavernous malformation in the left temporal lobe (arrow).

RCVS: reversible cerebral vasoconstriction syndrome; CTA: computed tomography angiography; MRI: magnetic resonance imaging.

had RCVS (8). Our results validate and extend these observations. While $84 \%$ of dissections in the French study involved the vertebral arteries, in our series $76 \%$ involved the extracranial carotid artery, consistent with the known ratio of carotid:vertebral artery involvement in cervical artery dissections (14). We found no association between dissection and RCVS complications such as infarcts or hemorrhage. The timing and mechanism of dissection remain uncertain. It is conceivable that vasoconstriction-induced changes in systemic blood pressure or arterial tone culminate in arterial dissection. Alternately, although RCVS is a reversible non-inflammatory arteriopathy with no abnormalities noted on histology or electron microscopy (6), ultrastructural abnormalities affecting the collagen or elastic lamina in the arterial wall may predispose to both vasoconstriction as well as dissection.

The high rate (nearly 13\%) of unruptured aneurysms in the RCVS group is notable. Unruptured aneurysms have been associated with cerebral vasoconstriction in multiple case reports $(7,10,11,15)$. In a prospective study of 67 RCVS patients, four had small $(2 \mathrm{~mm}-$ $3.8 \mathrm{~mm}$ ) unruptured aneurysms (4). In a study of 249 patients with Moyamoya disease (16), another noninflammatory cerebral arteriopathy, only $3.6 \%$ proved to have unruptured aneurysms. In our study, the PACNS group did not have saccular aneurysms although it is conceivable that small aneurysms, if present, were considered within the range of expected vasculitic abnormalities and not reported separately by the radiologist. The rate of unruptured aneurysms in the non-arteriopathy control group was $7.9 \%$, or $3.4 \%$ for aneurysms $>2 \mathrm{~mm}$, which is comparable to the $5 \%$ rate observed in prior studies (17). The pathophysiological relationship between RCVS and unruptured aneurysms is uncertain. Since RCVS is an acute syndrome, and our patients were generally imaged within one to two days after symptom onset, it is unlikely that these aneurysms resulted from chronic flow-related changes as postulated in Moyamoya or other arteriopathies. We have previously hypothesized that primary or vasoactive drug-induced abnormalities of vessel tone may explain areas of segmental vasodilation observed in RCVS (18) and perhaps contribute to aneurysm formation (10), similar to observations in vasoactive drug-associated ischemic stroke (19). In our study, approximately $60 \%$ of RCVS patients had prior exposure to vasoactive drugs. Unfortunately we do not have information about the dose and duration of exposure, or similar data in the other two groups, to study this issue further. Finally, as with dissections, it is possible that ultrastructural vessel wall abnormalities predispose to aneurysm formation.

The frequency of other vascular malformations appears to be high in the RCVS group; however, the combined rate of such lesions in the general population is not well studied. Our analysis was limited to the cerebral vasculature, so we cannot rule out a higher rate of systemic vascular abnormalities that are being reported with increasing frequency (20-22). Nevertheless, the heterogeneous anatomy of all these lesions suggests that there may be subtle ultrastructural or genetic abnormalities that lower the threshold for vasoconstriction, rather than vasoconstriction itself leading to the 
formation of vascular lesions such as dissection or aneurysms, for example, via blood pressure alterations.

Our study has several strengths and limitations. We present the first systematic controlled study of coexisting vascular lesions, and this is one of the largest case series of RCVS as well as PACNS. The more-sensitive angiographic modalities (DSA and CTA) were available for analysis in approximately $90 \%$, and brain MRI available in nearly all patients with RCVS and PACNS. However the differences in sensitivities of the different imaging modalities, improvement in their sensitivities over the study time span, and the lack of a standardized protocol for all three groups, are limitations. Study bias was limited because we included consecutive patients in the RCVS and non-arteriopathy groups, and used many resources to gather the PACNS group. Moreover, we analyzed the reports of clinically indicated imaging studies, so there was no bias in determining the rate of coexisting or incidental abnormalities. However, it is conceivable that some vascular lesions were not formally reported. The groups were imbalanced, e.g. controls were not agesex matched. However, RCVS remained a significant predictor in regression analysis including imaging modality, age, and gender, and the non-arteriopathy controls were older and also symptomatic, which if anything would favor a negative result.
The management of coexisting vascular abnormalities warrants discussion. It is unlikely that the vascular lesions cause the severe, explosive, recurrent headaches that characterize RCVS. While cervical artery dissection increases ischemic stroke risk especially in the first few days after onset, the risk of brain hemorrhage is also high in the first one to two weeks after the onset of RCVS. Hence, the use of antiplatelets and antithrombotic agents (to prevent ischemic stroke) should be individualized. Unruptured aneurysms are unlikely to explain the recurrent thunderclap headaches or angiographic narrowings that are so characteristic of RCVS, and to date have not culminated in rupture during an episode of RCVS. Hence, urgent intervention (clipping, coiling) is probably not warranted. However, aneurysms should be followed over time and treated as per current guidelines (23). Similarly, DVAs, cavernomas, and other vascular abnormalities need not be treated in the acute stage, but followed and managed long term as appropriate. Finally, although our results document a high rate of coexisting vascular lesions, these lesions are generally asymptomatic and the ones requiring intervention or follow-up are typically apparent on the initial vascular imaging studies in RCVS patients. Hence we do not advocate additional brain and systemic vascular imaging to specifically uncover such lesions.

\section{Clinical implications}

1. Patients with reversible cerebral vasoconstriction syndrome (RCVS) have a high prevalence of coexisting neurovascular abnormalities such as cervical artery dissection, unruptured aneurysms, and cavernomas.

2. These coexisting vascular abnormalities are typically asymptomatic and are not associated with complications and clinical outcomes after RCVS.

3. Their presence suggests that subtle ultrastructural arterial wall abnormalities may underlie their development and also predispose to RCVS.

\section{Declaration of conflicting interests}

The authors declared no potential conflicts of interest with respect to the research, authorship, and/or publication of this article.

\section{Funding}

The authors received no financial support for the research, authorship, and/or publication of this article.

\section{References}

1. Singhal AB, Hajj-Ali RA, Topcuoglu MA, et al. Reversible cerebral vasoconstriction syndromes: Analysis of 139 cases. Arch Neurol 2011; 68: 1005-1012.

2. Calabrese LH, Dodick DW, Schwedt TJ, et al. Narrative review: Reversible cerebral vasoconstriction syndromes. Ann Intern Med 2007; 146: 34-44.
3. Ducros A. Reversible cerebral vasoconstriction syndrome. Lancet Neurol 2012; 11: 906-917.

4. Ducros A, Boukobza M, Porcher R, et al. The clinical and radiological spectrum of reversible cerebral vasoconstriction syndrome. A prospective series of 67 patients. Brain 2007; 130: 3091-3101.

5. Chen SP, Fuh JL, Wang SJ, et al. Magnetic resonance angiography in reversible cerebral vasoconstriction syndromes. Ann Neurol 2010; 67: 648-656.

6. Singhal AB, Kimberly WT, Schaefer PW, et al. Case records of the Massachusetts General Hospital. Case 8-2009. A 36-year-old woman with headache, hypertension, and seizure 2 weeks post partum. N Engl J Med 2009; 360: 1126-1137.

7. Singhal AB. Postpartum angiopathy with reversible posterior leukoencephalopathy. Arch Neurol 2004; 61: 411-416. 
8. Mawet J, Boukobza M, Franc J, et al. Reversible cerebral vasoconstriction syndrome and cervical artery dissection in 20 patients. Neurology 2013; 81: 821-824.

9. Arnold M, Camus-Jacqmin M, Stapf C, et al. Postpartum cervicocephalic artery dissection. Stroke 2008; 39: 2377-2379.

10. Singhal AB. Thunderclap headache, reversible cerebral arterial vasoconstriction, and unruptured aneurysms. J Neurol Neurosurg Psychiatry 2002; 73: 96; author reply $96-97$.

11. Singhal AB, Caviness VS, Begleiter AF, et al. Cerebral vasoconstriction and stroke after use of serotonergic drugs. Neurology 2002; 58: 130-133.

12. Fugate JE, Ameriso SF, Ortiz G, et al. Variable presentations of postpartum angiopathy. Stroke 2012; 43: 670-676.

13. Hajj-Ali RA, Singhal AB, Benseler S, et al. Primary angiitis of the CNS. Lancet Neurol 2011; 10: 561-572.

14. Debette S, Grond-Ginsbach C, Bodenant M, et al. Differential features of carotid and vertebral artery dissections: The CADISP study. Neurology 2011; 77: 1174-1181.

15. Day JW and Raskin NH. Thunderclap headache: Symptom of unruptured cerebral aneurysm. Lancet 1986; 2: 1247-1248.

16. Yeon JY, Kim JS and Hong SC. Incidental major artery aneurysms in patients with non-hemorrhagic moyamoya disease. Acta Neurochir (Wien) 2011; 153: 1263-1270.
17. Harada K, Fukuyama K, Shirouzu T, et al. Prevalence of unruptured intracranial aneurysms in healthy asymptomatic Japanese adults: Differences in gender and age. Acta Neurochir (Wien) 2013; 155: 2037-2043.

18. Kobayashi M. Diffuse cerebrovascular dilation: Case report of amezinium metilsulfate-induced reversible cerebral vasoconstriction syndrome. Cephalalgia 2016; 36: 289-293.

19. McEvoy AW, Kitchen ND and Thomas DG. Intracerebral haemorrhage caused by drug abuse. Lancet 1998; 351: 1029.

20. Mukerji SS, Buchbinder BR and Singhal AB. Reversible cerebral vasoconstriction syndrome with reversible renal artery stenosis. Neurology 2015; 85: 201-202.

21. John S, Hajj-Ali RA, Min D, et al. Reversible cerebral vasoconstriction syndrome: Is it more than just cerebral vasoconstriction? Cephalalgia 2015; 35: 631-634.

22. Nouh A, Ruland S, Schneck MJ, et al. Reversible cerebral vasoconstriction syndrome with multivessel cervical artery dissections and a double aortic arch. J Stroke Cerebrovasc Dis 2014; 23: e141-e143.

23. Thompson BG, Brown RD Jr, Amin-Hanjani S, et al. Guidelines for the Management of Patients With Unruptured Intracranial Aneurysms: A Guideline for Healthcare Professionals From the American Heart Association/ American Stroke Association. Stroke 2015; 46: 2368-2400. 